\title{
A quantum Monte Carlo and density functional theory study of the electronic structure of peroxynitrite anion
}

\author{
J. A. W. Harkless ${ }^{a)}$ \\ Department of Chemistry, University of California at Berkeley, Berkeley, California 94720-1460 and \\ Chemical Sciences Division, Lawrence Berkeley National Laboratory, Berkeley, California 94720 \\ J. H. Rodriguez \\ Department of Physics, Purdue University, West Lafayette, Indiana 47907-1396 \\ L. Mitas \\ Department of Physics, North Carolina State University, Raleigh, North Carolina 27695-8202 \\ W. A. Lester, Jr. \\ Department of Chemistry, University of California at Berkeley, Berkeley, California 94720-1460 and \\ Chemical Sciences Division, Lawrence Berkeley National Laboratory, Berkeley, California 94720
}

(Received 16 April 2002; accepted 17 December 2002)

\begin{abstract}
Single point calculations of the ground state electronic structure of peroxynitrite anion have been performed at the optimized cis geometry using the restricted Hartree-Fock (RHF), Møller Plesset second order perturbation theory (MP2), generalized gradient approximation density functional theory (GGA DFT) in the B3LYP form and two quantum Monte Carlo (QMC) methods, variational Monte Carlo (VMC) and diffusion Monte Carlo (DMC). These calculations reveal differences in atomization energies estimated by B3LYP $(287.03 \mathrm{kcal} / \mathrm{mol})$, MP2 $(290.84 \mathrm{kcal} / \mathrm{mol})$, and DMC, $307.4(1.9) \mathrm{kcal} / \mathrm{mol}$, as compared to experiment, 313(1) $\mathrm{kcal} / \mathrm{mol}$. The error associated with MP2 and B3LYP methods is attributed largely to differential recovery of correlation energies for neutral nitrogen and oxygen atoms relative to the oxygen and peroxynitrite anions. In addition, basis set studies were carried out to determine potential sources of error in MP2 and B3LYP valence energy values. Our studies indicate that MP2 and B3LYP valence energies are strongly dependent on the presence of diffuse functions for the negative ions $\mathrm{O}^{-}$and $\mathrm{ONOO}^{-}$. (C) 2003 American Institute of Physics. [DOI: 10.1063/1.1544732]
\end{abstract}

\section{INTRODUCTION}

Peroxynitrite $\left(\mathrm{ONOO}^{-}\right)$is formed by the direct and rapid reaction of nitric oxide with superoxide anion. ${ }^{1-3} \mathrm{Be}-$ cause $\mathrm{ONOO}^{-}$can act as a one-electron oxidant or a twoelectron oxidant, it is able to react with a number of biomolecules including proteins, ${ }^{4-11}$ DNA, ${ }^{12,13}$ lipids, ${ }^{14}$ and antioxidants. ${ }^{15,16}$ The reactivity profile of peroxynitrite suggests that it is capable of crossing biological membranes faster than its known decomposition rate. ${ }^{17}$ Studies also suggest that production of peroxynitrite plays a pivotal role in the apoptosis (programmed cell death) of neural cells ${ }^{18}$ and aortic smooth muscle cells in rats. ${ }^{19}$ Similarly, peroxinitrite has been shown to induce apoptosis in human leukemia cells ${ }^{20,21}$ with clear dependence on its length of exposure and concentration. The same study also suggested the ineffectiveness of peroxynitrite at inducing apoptosis in some normal endothelial human cells. ${ }^{20}$ The high reactivity of peroxynitrite and its potential role in a wide variety of human diseases have stimulated our present study.

\footnotetext{
a) Bell Labs Cooperative Research Fellow at the University of California, Berkeley. Present address: Physical and Chemical Properties Division, National Institute of Standards and Technology, 100 Bureau Drive, Stop 8380, Gaithersburg, Maryland 20899-8380.
}

Theoretical studies of peroxynitrite ${ }^{22-25}$ have given some insight on its geometry and electronic structure. Some properties of this anion have also been inferred from studies of ONOOM $(\mathrm{M}=\mathrm{Li}, \mathrm{Na}, \mathrm{K})$ (Ref. 26) or decomposition of ONOOH. ${ }^{27}$ None of these studies focus on direct calculations of the molecule as it is formed in vivo, in its unattached ionic state. Factors that may account for the limited application of computational methods to this system are difficulties encountered with some basis set $a b$ initio methods in treating negative ions of molecules containing nitrogen and oxygen atoms. Motivated by success with quantum Monte Carlo (QMC) in the diffusion Monte Carlo (DMC) approach in treating negative ions ${ }^{28-30}$ and, in particular, accurate determinations of atomization energies of molecular systems, ${ }^{31-34}$ we undertook the present study to characterize more fully this important system of biological interest.

The remainder of the paper is organized as follows: Section II presents a brief discussion of DMC to highlight the basic ideas of the method; Sec. III describes the equilibrium geometry and some aspects of the electronic structure of $\mathrm{ONOO}^{-}$; Sec. IV presents valence and atomization energies obtained with various basis sets and methods; and Secs. V and VI discuss the results with some commentary on potential sources of error. 


\section{COMPUTATIONAL METHODS}

\section{A. Variational Monte Carlo (VMC)}

There is a similarity between the Schrödinger equation in imaginary time $(\tau=\iota t)$, written in atomic units,

$$
\frac{\partial \Phi}{\partial \tau}=\frac{1}{2} \nabla^{2} \Phi+V \Phi
$$

and a classical diffusion equation with a rate term,

$$
\frac{d C}{d t}=D \nabla^{2} C+k C .
$$

In Eq. (1), $\Phi$ is a quantum mechanical wave function, and $\nabla^{2}$ and $\mathrm{V}$ are the kinetic and potential energy operators, respectively. In Eq. (2), $C$ is the concentration, $D$ is the diffusion constant, and $k$ is a rate constant. When the quantum mechanical wave function in Eq. (1), serves as a probability density in imaginary time, no real time dynamics can be obtained. ${ }^{35-39}$ Using $\Phi$ in this manner is analogous to considering it as a concentration of electrons undergoing rate and diffusion processes.

The conceptually simple variational Monte Carlo (VMC) method $^{40-44}$ uses Monte Carlo integration to evaluate a trial function. Unlike basis set expansion ab initio methods which rely on accurate integration of molecular integrals to achieve high accuracy, VMC trial functions may be of any form. In atomic and molecular applications, this trial function is often a product of an independent particle wave function typically computed in one of the following approximations: HartreeFock (HF), configuration interaction (CI), or complete active space self-consistent field (CASSCF), and a correlation function that is explicitly dependent on interparticle distances. The probability distribution used in the Monte Carlo integration is taken as the square modulus of the trial function, and in all-electron VMC the energy is an upper bound to the exact energy of the state being evaluated. The form of the estimator used in VMC is

$$
\langle E\rangle=\frac{\langle\Psi|H| \Psi\rangle}{\langle\Psi \mid \Psi\rangle}=\frac{\left\langle\Psi\left|\frac{H \Psi}{\Psi}\right| \Psi\right\rangle}{\langle\Psi \mid \Psi\rangle}=\frac{\left\langle\Psi\left|E_{L}\right| \Psi\right\rangle}{\langle\Psi \mid \Psi\rangle}=\left\langle E_{L}\right\rangle,
$$

where $E_{L}$ is the "local energy," defined as $\mathrm{H} \Psi(\mathbf{B}) / \Psi(\mathbf{R})$.

\section{B. Diffusion Monte Carlo}

The DMC method is based on stochastic simulation of the Schrödinger equation in imaginary time; the latter may be written, ${ }^{45-47}$

$$
\frac{\partial f}{\partial t}=\frac{1}{2} \nabla(\nabla-F) f-\left(E_{L}-E_{T}\right) f,
$$

where the distribution

$$
f \equiv \Psi_{T} \Phi \text {. }
$$

Here $\Psi_{T}$ is a known wave function and $\Phi$ is the exact solution, and

$$
F \equiv \ln \left|\Psi_{T}\right|^{2}
$$

is labeled the quantum force. The parameter $E_{T}$ is an energy offset, and

$$
E_{L}(\mathbf{R}) \equiv \frac{\hat{H} \Psi_{T}(\mathbf{R})}{\Psi_{T}(\mathbf{R})}
$$

is the local energy, where $\mathbf{R}$ denotes the $3 N$ coordinates of the system. We choose $\Psi_{T}$ as the product of an independentparticle wave function, $\Psi_{0}$, and a correlation function $U$, i.e.,

$$
\Psi_{T}=\Psi_{0} \exp U
$$

where

$$
U \equiv U\left(r_{i j}, r_{i \alpha}, r_{i \beta}\right) .
$$

In Eq. (9), the $r_{i j}$ are electron-electron distances, and the $r_{i \alpha}$ and $r_{i \beta}$ are electron-nuclear distances. The parameter $E_{T}$ is generally chosen to be close to the expected value of the local energy in order to reduce the magnitude of the last term on the r.h.s. of Eq. (4). The function $F$ produces a drift in the simulation that increases sampling in the regions where the wave function is large. The fixed node approximation imposes the nodal structure of $\Psi_{T}$ on $\Phi$ which insures $f$ is non-negative for all $\mathbf{R}$.

In the present study, the independent particle wave function $\Psi_{0}$ is chosen as a restricted Hartree-Fock (RHF) wave function. The correlation function is a Schmidt-Moskowitz ${ }^{44}$ adaptation of the Boys-Handy function, ${ }^{48}$ which includes terms describing two- and three-body interactions. The correlation function parameters were optimized by minimizing the variance of the energy estimate from a Monte Carlo integration.

There are a number of DMC studies that have demonstrated the successful application of this method in conjunction with effective core potentials (ECP-DMC) ${ }^{49-54}$ More specifically, ECP-DMC studies of second row bond energies and electron affinities have been reported which agree well with experiment ${ }^{54,55}$ and have been able to distinguish between alternative experimental analyses. ${ }^{56}$ The DMC method is relatively insensitive to choice of basis set for the independent-particle part of the trial function. With a singlereference wave function, we used a cc-pVTZ basis set ${ }^{57}$ with no $F$ functions for single-point energy calculations. In DMC, effective sampling of the regions of space occupied by core electrons is costly; it is this additional cost that serves as additional motivation for using ECP's. Estimates of computational effort for all-electron QMC for atoms yield ${ }^{55-65}$ dependence, where $Z$ is the atomic number. ${ }^{50}$ The use of ECP's has been found to reduce this dependence to roughly $N^{3}$, where $N$ is the effective atomic number, or stated more directly, the number of electrons to be simulated. ${ }^{50}$ To provide a more concrete and recent example, we turn to Ref. 31 . Table VI of this paper compares the CPU and disk storage requirements of LDA, G2 theory, and DMC calculations, as well as the parallelism and scaling. One finds that LDA is at least an order of magnitude less costly than DMC and G2, and that $\mathrm{G} 2$ requires roughly three orders of magnitude more disk storage than LDA and DMC. Timing estimates are subject to variances owing to machine configuration and other system-specific features. 
TABLE I. Optimized bond lengths (angstroms) and angles (degrees) of $\mathrm{ONOO}^{-}$

\begin{tabular}{lccc}
\hline \hline & $\begin{array}{c}\text { B3LYP } \\
6-311+\mathrm{G}^{*}\end{array}$ & $\begin{array}{c}\text { B3LYP } \\
\text { cc-pVTZ }\end{array}$ & $\begin{array}{c}\text { MP2 } \\
\text { cc-pVTZ }\end{array}$ \\
\hline $\mathrm{O} 1=\mathrm{N} 1$ & 1.213 & 1.214 & 1.234 \\
$\mathrm{~N} 1-\mathrm{O} 2$ & 1.367 & 1.362 & 1.336 \\
$\mathrm{O} 2-\mathrm{O} 3$ & 1.388 & 1.382 & 1.358 \\
$\mathrm{O} 1-\mathrm{N} 1-\mathrm{O} 2$ & 116.3 & 116.1 & 115.4 \\
$\mathrm{~N} 1-\mathrm{O} 2-\mathrm{O} 3$ & 118.4 & 118.4 & 117.9 \\
\hline \hline
\end{tabular}

In addition to the benefits of ECPs in general, there are alternative ECPs that have been recently developed for the oxygen and nitrogen atoms which enhance the efficiency of DMC simulations. These ECPs, which are similar to the soft ECPs of condensed matter physics ${ }^{58}$ and to a soft ECP reported for carbon, ${ }^{59}$ are characterized by the absence of the $r^{-2}$ term common to ECPs in quantum chemistry. ${ }^{59,60} \mathrm{DMC}$ simulations using the soft ECP are found to yield a smaller statistical error bar than the Stevens, Basch, and Krauss (SBK) function ${ }^{60}$ for comparable computing time. The soft ECP also yields comparable valence variational Monte carlo (VMC) energies and slightly lower valence DMC energies, compared to the SBK ECP. For these reasons, we carried out DMC calculations only using soft ECPs.

\section{GEOMETRY AND VALENCE ELECTRONIC STRUCTURE}

The geometry of $\mathrm{ONOO}^{-}$was optimized at the B3LYP/6-311+G*, B3LYP/cc-pVTZ, and MP2/cc-pVTZ levels. The B3LYP parameters obtained with $6-311+\mathrm{G}^{*}(92$ basis functions) and cc-pVTZ (140 basis functions) were very close and displayed similar trends as those derived from MP2; see Table I. In agreement with previous studies, ${ }^{22,23}$ our optimizations revealed the planar cis conformer as the most stable geometry with outer $\mathrm{O} 1=\mathrm{N} 1$, central $\mathrm{N} 1-\mathrm{O} 2$, and outer $\mathrm{O} 2-\mathrm{O} 3$ bonds (Fig. 1).

Figure 2 shows the frontier Kohn-Sham orbitals of $\mathrm{ONOO}^{-}$. The HOMO is mainly localized on the terminal oxygen of the superoxide fragment. By contrast, the LUMO is mainly localized on the nitric oxide fragment and in this regard is analogous to the $\pi^{*}$ orbitals of free NO. ${ }^{61}$ To check the nature of the singlet ground state of $\mathrm{ONOO}^{-}$both restricted and unrestricted density functional calculations were carried out. The frontier orbitals with $\alpha$ and $\beta$ spin index were indistinguishable in the unrestricted calculation con-

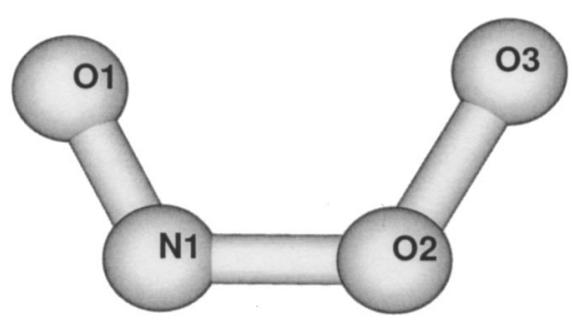

FIG. 1. Optimized geometry of peroxynitrite ion. Metric parameters are given in Table I.
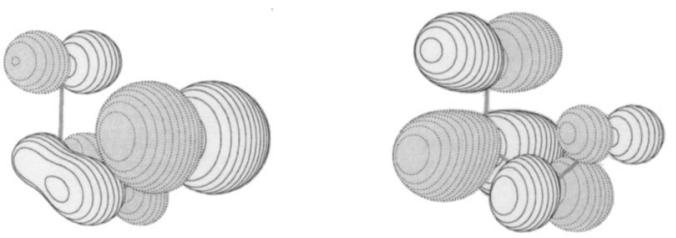

FIG. 2. HOMO (left) and LUMO (right) of peroxynitrite calculated at the B3LYP/cc-pVTZ level. The HOMO is mainly localized on the superoxide fragment, whereas the LUMO is mainly localized on the nitric oxide fragment.

firming the closed-shell singlet character of the $\mathrm{ONOO}^{-}$ ground state. We also notice that, despite the auxiliary character of Kohn-Sham orbitals, ${ }^{62}$ which makes it difficult to give them a well defined physical meaning, DFT and $a b$ initio wave function methods (MP2) yielded similar trends in frontier orbital composition and charge distribution. As shown in Table II, most of the negative charge is localized on the terminal oxygen of the superoxide fragment (O3), consistent with the spatial distribution of the HOMO. The peroxynitrite anion is known to react with transition metalcontaining complexes, for example with the irons of hemoproteins. ${ }^{63}$ The similarity of its LUMO with the $\pi^{*}$ orbitals of free NO strongly suggests that some similar metal-ligand bonding interactions are at work in the binding to metals of these two ligands. ${ }^{64}$

\section{CALCULATED ENERGIES}

For the QMC calculations, we used RHF/cc-pVTZ orbitals generated at the B3LYP/cc-pVTZ optimized geometry. ${ }^{57,65}$ Ground state energy calculations with each of the above methods at their respective optimized structures revealed a specific ordering of total valence energies calculated by RHF, MP2, and B3LYP, as well as VMC and DMC. The ordering of the energies in Table III is typical for this group of methods.

Although the VMC and DMC calculations used RHF orbitals with a cc-pVTZ basis, the QMC results only have a small, indirect basis set dependence. Basis set effects in DMC are limited to instances of poor description of the orbitals from traditional $a b$ initio techniques. In order to guarantee effective orbital descriptions, we used either large basis sets or custom bases that have been developed to work well in DMC calculations. We also included a VMC valence energy for the SBK-ECP for comparison with a soft ECP developed by one of us (L.M.). As mentioned above, the soft ECPs are more stable and provide slightly lower energies. The increased efficiency of the soft ECP can be observed by comparing the error bars of the VMC calculations: 0.0025

TABLE II. NBO atomic charge densities of $\mathrm{ONOO}^{-}$(cc-pVTZ basis).

\begin{tabular}{lcc}
\hline \hline & B3LYP & MP2 \\
\hline O1 & -0.457 & -0.419 \\
N1 & +0.196 & +0.174 \\
O2 & -0.142 & -0.087 \\
O3 & -0.598 & -0.667 \\
\hline \hline
\end{tabular}


TABLE III. Valence energies of $\mathrm{ONOO}^{-}$(hartree).

\begin{tabular}{llll}
\hline \hline \multicolumn{1}{c}{ Method } & \multicolumn{1}{c}{$6-311+\mathrm{G}^{*}$} & \multicolumn{1}{c}{ cc-pVTZ } & aug-cc-pVTZ \\
\hline RHF & -56.67029 & -56.71748 & -56.73253 \\
MP2 & -57.48405 & -57.65572 & -57.70277 \\
B3LYP & -57.82000 & -57.85368 & -57.87557 \\
VMC/SBK ECP & & $-57.5005(25)^{\mathrm{a}}$ & \\
VMC/soft ECP & & $-57.5111(10)^{\mathrm{a}}$ & \\
DMC/soft ECP & & $-57.8509(9)^{\mathrm{a}}$ & \\
\hline \hline
\end{tabular}

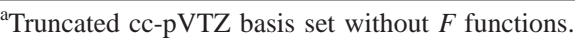

Hartree (SBK) and 0.0010 Hartree (soft). These statistics are for equivalent computer time; halving the error in the VMC calculation with the SBK ECPs would require about four times more computational effort. The increased efficiency is the reason we restricted use to only the soft ECPs in the DMC calculations. Soft ECPs suitable for QMC have recently been extended to Be through $\mathrm{Ne}$ and $\mathrm{Al}$ through $\mathrm{Ar}^{66}$

We used the following expression to estimate the atomization energy $\left(E_{a}\right)$ of peroxynitrite in VMC and DMC,

$$
E_{a}=E\left(\mathrm{O}^{-}\right)+2 E(\mathrm{O})+E(\mathrm{~N})-\left[E\left(\mathrm{ONOO}^{-}\right)-\mathrm{ZPE}\right],
$$

where $E$ is the Monte Carlo valence energy of the indicated species, and ZPE is the zero-point vibrational energy determined by theory. We used an identical construction for the atomization energy estimates in the all-electron calculations for B3LYP, MP2, and RHF. While we computed energies for all species with and without the use of ECP's in the RHF, MP2, and B3LYP methods, we report the all-electron values in Table IV. The "valence" values reported in the other tables incorporate the ECP's of Stevens, Basch, and Krauss, ${ }^{60}$ and are included as such to facilitate comparison to our ECP Monte Carlo energies. We find that the oxygen anion is a reasonable choice for the charged atomic species because it is more electronegative than nitrogen, and because of our use of the electron affinity of oxygen in computing an experimental atomization energy. This decision is also supported by examination of the results from the basis set methods, which consistently placed partial negative charges on the oxygens independent of the level of theory or basis set used (Table II). The experimental atomization energy was estimated by taking the difference of the heats of formation for the three atomic species from standard reference tables ${ }^{67}$ and the heat of formation reported by Mereyni et al. ${ }^{68}$ As shown in Table IV, atomization energies obtained with RHF,

TABLE IV. Atomization energies of $\mathrm{ONOO}^{-}(\mathrm{kcal} / \mathrm{mol})$.

\begin{tabular}{lccc}
\hline \hline \multicolumn{1}{c}{ Method } & 6-311+G* & cc-pVTZ & aug-cc-pVTZ \\
\hline All-electron RHF & 62.65 & 78.19 & 69.63 \\
All-electron MP2 & 267.11 & 290.84 & 282.99 \\
All-electron B3LYP & 286.01 & 287.03 & 276.02 \\
VMC/soft ECP & & $204.0(1.8)^{\mathrm{a}}$ & \\
DMC/soft ECP & & $307.4(1.9)^{\mathrm{a}}$ & \\
Experiment $^{\mathrm{b}}$ & & $313(1)$ & \\
\hline \hline
\end{tabular}

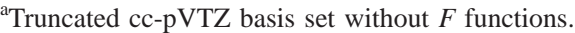

${ }^{\mathrm{b}}$ References 67 and 68 .
TABLE V. Valence energies of $\mathrm{O}^{-}$(hartree).

\begin{tabular}{lcccr}
\hline \hline Method & 6-311G* & 6-311+G* & cc-pVTZ & aug-cc-pVTZ \\
\hline ROHF & -15.59000 & -15.62796 & -15.61795 & -15.64132 \\
MP2 & -15.75216 & -15.81027 & -15.82570 & -15.86812 \\
B3LYP & -15.85635 & -15.91733 & -15.89380 & -15.93265 \\
VMC & & $-15.8233(5)^{\mathrm{a}}$ & \\
DMC & & $-15.8854(8)^{\mathrm{a}}$ & \\
\hline \hline
\end{tabular}

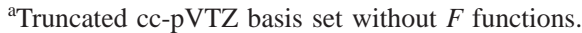

MP2, B3LYP, and VMC yield estimates in poor accord with the experimental atomization energy. Good agreement, however, is obtained with DMC.

\section{DISCUSSION}

Calculated oxygen anion valence energies provide the clearest indicator of a potential source of error in estimating the atomization energy from ab initio wave function and GGA DFT methods for the present systems. Changing from the $6-311+\mathrm{G}^{*}$ (92 basis functions) to the larger cc-pVTZ (142 basis functions) unexpectedly decreased the valence energies calculated with ROHF and B3LYP (Table V). The generally expected effect of increasing the number of basis functions is a decrease in energy due to an improved ability to approximate the wave function of a given system. This unexpected basis set effect encountered in ROHF and B3LYP energies led us to investigate the specific role of the types, as opposed to the number, of basis functions. More specifically, additional calculations were performed using the 6-311G* and aug-cc-pVTZ bases to complete Table V and to investigate the effect of diffuse functions on the converged valence energies of $\mathrm{O}^{-}$and $\mathrm{ONOO}^{-}$.

In Table VI we compare valence energy differences from calculations with the $6-311 \mathrm{G}^{*}$ and $6-311+\mathrm{G}^{*}$ split-valence bases, and calculations with the cc-pVTZ and aug-cc-pVTZ bases. With the additional diffuse functions, the ROHF (B3LYP) energies for the oxygen anion in the split-valence bases were lowered by 0.039 (0.057) Hartree, whereas the peroxynitrite energies were lowered by $0.021(0.033)$ Hartree. For the correlation consistent bases, oxygen anion energies decreased by $0.024(0.038)$ Hartree and peroxynitrite energies by 0.014 (0.027) Hartree. Upon addition of diffuse functions, the MP2 energy decreases were roughly equal for $\mathrm{O}^{-}$and $\mathrm{ONOO}^{-}$in the correlation consistent bases. These findings, however, are consistent with reports that stress the need for diffuse orbitals when treating negative ions. ${ }^{69,70}$ Even though single point $6-311+\mathrm{G}^{*}$ calculations on

TABLE VI. Change in anion electronic energies due to addition of diffuse functions (hartree). $\Delta \mathrm{E}_{\mathrm{cc}}=\mathrm{E}_{\text {aug-cc-pvTZ }}-\mathrm{E}_{\mathrm{cc}-\mathrm{pVTZ}}{ }^{\mathrm{a}}$ and $\Delta \mathrm{E}_{\mathrm{sv}}=\mathrm{E}_{6-311+\mathrm{G} *}$ $-\mathrm{E}_{6-311 \mathrm{G} *}$ b $^{\mathrm{b}}$

\begin{tabular}{lcccc}
\hline \hline & $\Delta \mathrm{E}_{\mathrm{sv}}, \mathrm{O}^{-}$ & $\Delta \mathrm{E}_{\mathrm{cc}}, \mathrm{O}^{-}$ & $\Delta \mathrm{E}_{\mathrm{sv}}, \mathrm{ONOO}^{-}$ & $\Delta \mathrm{E}_{\mathrm{cc}}, \mathrm{ONOO}^{-}$ \\
\hline RHF & -0.038554 & -0.023829 & -0.021175 & -0.014166 \\
MP2 & -0.055501 & -0.043883 & -0.038974 & -0.046126 \\
B3LYP & -0.057427 & -0.037638 & -0.032575 & -0.026744 \\
\hline \hline
\end{tabular}

aThe "cc" subscript refers to "correlation consistent."

'The "sv" subscript refers to "split-valence." 
TABLE VII. Valence energies of oxygen and nitrogen atoms (hartree).

\begin{tabular}{lcccc}
\hline \hline & \multicolumn{2}{c}{$\mathrm{O}$} & \multicolumn{2}{c}{$\mathrm{N}$} \\
& $6-311+\mathrm{G}^{*}$ & cc-pVTZ & $6-311+\mathrm{G}^{*}$ & cc-pVTZ \\
\cline { 2 - 5 } ROHF & -15.64647 & -15.65863 & -9.63054 & -9.63793 \\
MP2 & -15.77297 & -15.81226 & -9.71710 & -9.74070 \\
B3LYP & -15.86273 & -15.87323 & -9.76539 & -9.77185 \\
VMC & $-15.8067(6)^{\mathrm{a}}$ & $-9.7394(2)^{\mathrm{a}}$ \\
DMC & $-15.8524(4)^{\mathrm{a}}$ & $-9.7613(5)^{\mathrm{a}}$ \\
\hline
\end{tabular}

Truncated cc-pVTZ basis set without $F$ functions.

$\mathrm{ONOO}^{-}$included a smaller number of basis functions as compared to cc-pVTZ, our results strongly suggest that it is the presence of diffuse functions in the former basis set that mainly accounts for the improved energies of the anions studied. In fact, for neutral oxygen and nitrogen the energies were slightly lower for cc-pVTZ relative to $6-311+\mathrm{G}^{*}$. This observation is also consistent with studies that report little effect of diffuse functions on some neutral molecules. ${ }^{70}$ In addition, the energy differences due to basis set effects were consistent regardless of the presence of ECPs. Improvement due to diffuse functions differed in the third decimal place, with basis set effects being more pronounced in the valence calculations (Table VII).

\section{CONCLUSIONS}

The RHF, MP2, B3LYP DFT, and DMC methods have been used to calculate the atomization energy of peroxynitrite anion. The DMC results are found to lie closest-within $5 \mathrm{kcal} / \mathrm{mol}$ - to the experimental value. We also found that the use of soft ECPs instead of the traditional SBK-ECPs provided substantial benefit by improving the overall efficiency of VMC and DMC calculations.

Atomization energy estimates from B3LYP and MP2, even with large basis sets, were in poor agreement with the experimental value. At the cc-pVTZ level, the MP2 and B3LYP methods underestimate the experimental value by approximately $25 \mathrm{kcal} / \mathrm{mol}$ and $40 \mathrm{kcal} / \mathrm{mol}$, respectively. The atomization energy obtained with VMC was in particularly poor agreement. We attribute the underperformance of VMC to the use of a single-reference starting point for a system with multireference characteristics. At the same time, ROHF, MP2, and B3LYP valence energies of the oxygen anion showed a larger decrease than those of peroxynitrite upon addition of diffuse functions in the split-valence bases. This suggests that a main source of error when calculating atomization energies with these latter methods is the difference in improvement of the valence energies of the anions and atoms of Eq. (10) due to differences in levels of correlation energy recovered. Finally, our analysis of the valence energies reiterates the need for diffuse functions when treating negative ions such as $\mathrm{O}^{-}$and $\mathrm{ONOO}^{-}$.

\section{ACKNOWLEDGMENTS}

J.A.W.H. thanks Bell Labs for providing fellowship support. This work was supported by the Director, Office of Science, Office of Basic Energy Sciences, Chemical Sciences Division of the U.S. Department of Energy under Con- tract No. DE-AC03-76F00098, and by NSF Grant No. DMR0102668 (L.M.). Calculations were performed at the National Energy Research Scientific Computing Center (NERSC) and the National Center for Supercomputer Applications (NCSA).

${ }^{1}$ J. S. Beckman, J. Chen, P. A. Marshall, and B. A. Freeman, Proc. Natl. Acad. Sci. U.S.A. 87, 1620 (1990).

${ }^{2}$ R. E. Huie and S. Padmaja, Free Radical Res. Commun. 18, 195 (1993).

${ }^{3}$ N. V. Blqugh and O. C. Zafiriou, Inorg. Chem. 24, 3504 (1985).

${ }^{4}$ H. Ischiropoulos, L. Zhu, J. Chen, M. Tsai, J. C. Martin, C. D. Smith, and J. S. Beckman, Arch. Biochem. Biophys. 298, 431 (1992).

${ }^{5}$ J. S. Beckman, H. Ischiropoulos, L. Zhu, M. V. D. Woerd, C. Smith, J. Chen, J. Harrison, J. C. Martin, and M. Tsa, Arch. Biochem. Biophys. 298, 438 (1992).

${ }^{6}$ B. Alvarez, H. Rubbo, M. Kirk, S. Barnes, B. A. Freedman, and R. Radi, Chem. Res. Toxicol. 9, 390 (1996).

${ }^{7}$ S. Padmaja, M. S. Ramezanian, P. L. Bounds, and W. H. Koppenol, Redox. Rep. 2, 173 (1996).

${ }^{8}$ W. A. Pryor, X. Jin, and G. L. Squadrito, Proc. Natl. Acad. Sci. U.S.A. 91, 11173 (1994).

${ }^{9}$ S. Padmaja, G. L. Squadrito, and W. A. Pryor, Arch. Biochem. Biophys. 349, 1 (1998).

${ }^{10}$ R. Floris, R. Piersma, G. Yang, P. Jones, and R. Wever, Eur. J. Biochem. 215, 767 (1993).

${ }^{11}$ L. Castro, M. Rodriguez, and R. Radi, J. Biol. Chem. 269, 29409 (1994).

${ }^{12}$ P. A. King, V. E. Anderson, J. O. Edwards, G. Gustafson, R. C. Plumb, and J. W. Suggs, J. Am. Chem. Soc. 114, 5430 (1992).

${ }^{13}$ P. A. King, E. Jamison, D. Strahs, V. E. Anderson, and M. Brenowitz, Nucleic Acids Res. 21, 2473 (1993).

${ }^{14}$ R. Radi, J. S. Beckman, K. M. Bush, and B. A. Freeman, Arch. Biochem. Biophys. 288, 481 (1991).

${ }^{15}$ R. Radi, J. S. Beckman, K. M. Bush, and B. A. Freeman, J. Biol. Chem. 266, 4244 (1991).

${ }^{16}$ D. Bartlett, D. F. Church, P. L. Bounds, and W. H. Koppenol, Free Radic. Biol. Med. 18, 85 (1995).

${ }^{17}$ J. Lee, J. A. Hunt, and J. T. Groves, J. Am. Chem. Soc. 120, 7493 (1998).

${ }^{18}$ J. N. Keller, M. S. Kindy, F. W. Holtsberg, D. K. St. Clair, H. C. Yen, A. Germeyer, S. M. Steiner, A. J. Bruce-Keller, J. B. Hutchins, and M. P. Mattson, J. Neurosci. 18, 687 (1998).

${ }^{19}$ M. O'Connor, A. L. Salzman, and C. Szabo, Shock 8, 439 (1997).

${ }^{20}$ K.-T. Lin, J.-Y. Xue, M. Nomen, B. Spur, and P. Y.-K. Wong, J. Biol. Chem. 270, 16487 (1995)

${ }^{21}$ K.-T. Lin, J.-Y. Xue, F. F. Sun, and P. Y.-K. Wong, Biochem. Biophys. Res. Commun. 230, 115 (1997).

${ }^{22}$ M. Krauss, Chem. Phys. Lett. 222, 513 (1994).

${ }^{23}$ H.-H. Tsai, T. P. Hamilton, J.-H. M. Tsai, J. G. Harrison, and J. S. Beckman, J. Phys. Chem. 100, 6942 (1996).

${ }^{24}$ G. V. Shustov, R. Spinney, and A. Rauk, J. Am. Chem. Soc. 122, 1191 (2000).

${ }^{25}$ M. D. Bartberger, L. P. Olson, and K. N. Houk, J. Am. Chem. Soc. 11, 710 (1998).

${ }^{26}$ H.-H. Tsai, T. P. Hamilton, J.-H. M. Tsai, J. G. Harrison, and J. S. Beckman, J. Phys. Chem. 100, 6942 (1996).

${ }^{27} \mathrm{~J}$. D. Watts (personal communication).

${ }^{28}$ R. N. Barnett, P. J. Reynolds, and W. A. Lester, Jr., J. Chem. Phys. 84, 4992 (1986).

${ }^{29}$ T. Yoshida, Y. Mizushima, and K. Iguchi, J. Chem. Phys. 89, 5815 (1988).

${ }^{30}$ G. Morosi, M. Mella, and D. Bressanini, J. Chem. Phys. 111, 6755 (1999).

${ }^{31}$ J. C. Grossman, W. A. Lester, Jr., and S. G. Louie, J. Am. Chem. Soc. 122, 705 (2000).

${ }^{32}$ J. A. W. Harkless and W. A. Lester, Jr., J. Chem. Phys. 113, 2680 (2000).

${ }^{33}$ J. C. Grossman and L. Mitas, Phys. Rev. Lett. 79, 4353 (1997).

${ }^{34}$ C. W. Greeff and W. A. Lester, Jr., J. Chem. Phys. 106, 6412 (1997).

${ }^{35}$ J. B. Anderson, Int. Rev. Phys. Chem. 14, 14 (1995).

${ }^{36}$ K. Ragavachari and J. B. Anderson, J. Phys. Chem. 100, 12960 (1996).

${ }^{37}$ Recent Advances in Quantum Monte Carlo Methods, edited by W. A. Lester, Jr. (World Scientific, Singapore, 1997).

${ }^{38}$ D. M. Ceperley and L. Mitáš, Adv. Chem. Phys. XCIII, 1 (1997).

39 J. B. Anderson, Rev. Comput. Chem. 13, 133 (1999).

${ }^{40}$ R. E. Lowther and R. L. Coldwell, Phys. Rev. A 22, 14 (1980).

${ }^{41}$ S. Y. Huang, Z. Sun, and W. A. Lester, Jr., J. Chem. Phys. 92, 597 (1990).

${ }^{42}$ C. J. Umrigar, K. G. Wilson, and J. W. Wilkins, Phys. Rev. Lett. 60, 1719 
(1988); C. J. Umrigar, Int. J. Quantum Chem., Quantum Chem. Symp. 23, 217 (1989).

${ }^{43}$ J. W. Moskowitz and M. H. Kalos, Int. J. Quantum Chem. 20, 1107 (1981).

${ }^{44}$ K. E. Schmidt and J. W. Moskowitz, J. Chem. Phys. 93, 4172 (1990).

${ }^{45}$ B. L. Hammond, W. A. Lester, Jr., and P. J. Reynolds, Monte Carlo Methods in Ab Initio Quantum Chemistry (World Scientific, Singapore, 1994).

${ }^{46}$ D. M. Ceperley and L. Mitáš, Adv. Chem. Phys. XCIII, 1 (1996).

${ }^{47}$ J. B. Anderson, Rev. Comput. Chem. 13, 133 (1999).

${ }^{48}$ S. F. Boys and N. C. Handy, Proc. R. Soc. London, Ser. A 310, 63 (1969).

${ }^{49}$ M. M. Hurley and P. A. Christiansen, J. Chem. Phys. 86, 1069 (1987).

${ }^{50}$ B. L. Hammond, P. J. Reynolds, and W. A. Lester, Jr., J. Chem. Phys. 87, 1130 (1987)

${ }^{51}$ P. A. Christiansen, J. Chem. Phys. 88, 4867 (1988).

${ }^{52}$ P. A. Christiansen, J. Chem. Phys. 93, 6654 (1990).

${ }^{53}$ H. J. Flad, A. Savin, and H. Preuss, J. Chem. Phys. 97, 459 (1992).

${ }^{54}$ C. W. Greeff, W. A. Lester, Jr., and B. L. Hammond, in Recent Advances in Quantum Monte Carlo Methods, edited by W. A. Lester, Jr. (World Scientific, Singapore, 1997), p. 117.

${ }^{55}$ L. Mitás and J. C. Grossman, in Recent Advances in Quantum Monte Carlo Methods, edited by W. A. Lester, Jr. (World Scientific, Singapore, 1997), p. 133.

${ }^{56}$ C. W. Greeff and W. A. Lester, Jr., J. Chem. Phys. 106, 6412 (1997).

${ }^{57}$ T. H. Dunning, Jr., J. Chem. Phys. 90, 1007 (1989). Basis sets were obtained from the Extensible Computational Chemistry Environment Basis Set Database, as developed and distributed by the Molecular Science Computing Facility, Environmental and Molecular Sciences Laboratory which is part of the Pacific Northwest Laboratory, P.O. Box 999, Richland, Washington 99352, USA, and funded by the U.S. Department of Energy.
The Pacific Northwest Laboratory is a multiprogram laboratory operated by Battelle Memorial Institue for the U.S. Department of Energy under Contract No. DE-AC06-76RLO 1830. Contact David Feller or Karen Schuchardt for further information.

${ }^{58}$ T. Sasake, A. M. Rappe, and S. G. Louie, Sci. Rep. Res. Inst. Tohoku Univ. A 39, 37 (1993)

${ }^{59}$ C. W. Greeff and W. A. Lester, Jr., J. Chem. Phys. 109, 1607 (1998).

${ }^{60}$ W. J. Stevens, H. Basch, and M. Krauss, J. Chem. Phys. 81, 6026 (1984).

${ }^{61}$ J. H. Rodriguez, Y.-M. Xia, and P. G. Debrunner, J. Am. Chem. Soc. 121, 7846 (1999)

${ }^{62}$ R. G. Parr and W. Yang, Density Functional Theory of Atoms and Molecules (Clarendon, Oxford, 1989).

${ }^{63}$ D. Pietraforte, A. M. Salzano, G. Scorza, G. Marino, and M. Minetti, Biochemistry 40, 15300 (2001).

${ }^{64}$ NBO 4.0, E. D. Glendening, J. K. Badenhoop, A. E. Reed, J. E. Carpenter, and F. Weinhold (Theoretical Chemistry Institute, University of Wisconsin, Madison, 1996).

${ }^{65}$ M. J. Frisch, G. W. Trucks, H. B. Schlegel et al. Gaussian 94, Revision E.1, Gaussian, Inc., Pittsburgh, PA, 1995.

${ }^{66}$ I. Ovcharenko, A. Aspuru-Guzik, and W. A. Lester, Jr., J. Chem. Phys. 114, 7790 (2001).

${ }^{67}$ Handbook of Chemistry and Physics, 64th ed. (CRC, Boca Raton), pp. D-77, D-78, E-62.

${ }^{68}$ G. Merenyi, J. Lind, S. Goldstein, and G. Czapski, J. Phys. Chem. A 103, 5685 (1999)

${ }^{69}$ W. J. Hehre, L. Radom, P. v. R. Schleyer, and J. A. Pople, Ab Initio Molecular Orbital Theory (Wiley, New York, 1986).

${ }^{70}$ J. B. Foresman and A. Frisch, Exploring Chemistry with Electronic Structure Methods (Gaussian, Inc., Pittsburgh, Pennsylvania, 1993). 